Article

\title{
Determination of Optimum Frequency for Electromagnetic-Assisted Nanofluid Core Flooding
}

\author{
Fatai A. Wahaab *(D), Noorhana Yahya *, Afza Shafie, Hassan Soleimani, Amir Rostami and \\ Menaka Ganeson
}

Fundamental and Applied Sciences Department, Universiti Teknologi PETRONAS, Tronoh, Bandar Seri Iskandar, Perak Darul Ridzuan 32610, Malaysia; afza@utp.edu.my (A.A.); hassan.soleimani@utp.edu.my (H.S.); amir.rostami@utp.edu.my (A.R.); menaka.ganeson@utp.edu.my (M.G.)

* Correspondence: fatai_17007358@utp.edu.my (F.A.W.); noorhana_yahya@utp.edu.my (N.Y.)

Received: 3 August 2019; Accepted: 15 September 2019; Published: 29 October 2019

\begin{abstract}
The coupling of electromagnetic (EM) wave to nanoflooding experiments, termed EM-assisted nanoflooding has gained enormous attention in recent years. This brings about the consideration of transmitter efficiency and the propagation pattern of the ensuing EM wave, both of which depend on reservoir electromagnetic properties. However, this has not been considered in previous works, despite its potential to improve the efficiency of the process through energy maximization. Hence, this study considers the effects of reservoir EM properties on the operation of a transmitter. The EM properties of saturated reservoir rock samples were measured. Afterward, a half-wave dipole antenna was modeled in a cylindrical reservoir based on the measured EM properties. The EM propagation pattern of the antenna was simulated in a frequency range of $100 \mathrm{MHz}-2.0 \mathrm{GHz}$ using the finite element method. The antenna was found to display dual resonance (at $800 \mathrm{MHz}$ and $1.3 \mathrm{GHz}$ ) for sandstone saturated with oil, brine and nanofluid. Application of the EM wave at resonance frequency can help increase the efficiency of EM-assisted nanoflooding.
\end{abstract}

Keywords: electromagnetic wave; transmitter; reservoir electromagnetic properties; resonant frequency; nanofluid

\section{Introduction}

The electromagnetic (EM) assisted enhanced oil recovery (EOR) method has gained significant attention for the recovery of residual oil [1]. The transfer of EM energy to reservoir materials depends on the molecular composition, such as the dipolar and magnetic molecules having free or bound charges like electrons or ions [2-4]. Therefore, EM waves behave differently at different interfaces within the reservoir [5,6]. The propagating EM wave exerts forces on the molecules, which then align in a direction parallel to the external EM field [7-9]. Application of electromagnetic (EM) waves of certain frequency ranges has been used in nanofluid core flooding experiments for stimulating the recovery of residual oil after water flooding [10-14]. The potential efficiency of this method has been demonstrated by several experimental and numerical studies [8,15-22]. Different recovery mechanisms other than the common EM heating method have been reported recently. For instance, the application of a magnetic field gradient for the polarization of ferromagnetic nanofluid during nanoflooding experiments has been demonstrated [3]. A considerable recovery factor attributed to interfacial tension (IFT) reduction [23] and hyperthermia phenomena has also been reported with the application of EM waves (frequency range 500-1000 MHz) [11,24,25]. There have also been similar reports on the use of EM waves for wettability alteration, implying an improvement in reservoir oil production rate [11,16,25-27]. Currently, few research articles have been generated on transmitter design considerations for EM-assisted oil recovery processes. For instance, numerical 
studies on EM-assisted oil recovery using a shaped dipole antenna was conducted by [28], likewise, a cylindrical antenna model using the wavelets analysis method has also been reported [29]. Similarly, dimensional constraints have been considered in simulating three types of antennae at different operating frequencies, $915 \mathrm{MHz}, 2.45 \mathrm{GHz}$ and $5.8 \mathrm{GHz}$ [4].

Despite the amount of reported studies, the effect of reservoir EM properties at varying formation fluid contents on the efficiency of the EM transmitter, and its propagation pattern have not been considered. Most of the reported studies irradiate the reservoir with EM waves of an arbitrary frequency (sometimes based on dimensional constraints) without considering the medium's EM response, and its effect on the antenna operating frequency. Thus, this area requires further investigation. This is crucial because the operating frequency, penetration depth and radiation pattern of a transmitter depend on reservoir EM properties. These properties include the electrical conductivity $\sigma$, dielectric permittivity $\varepsilon$ and magnetic permeability $\mu$, all of which vary with fluid content, and are frequency dependent in varying degrees $[30,31]$. Consequently, it is vital to factor reservoir EM properties into the determination of antenna dimensions and operating frequency to achieve the desired uniform far-field pattern, homogenous EM energy dissipation and to avoid hot spots on the antenna surface [25]. Furthermore, the three-dimensional far-field pattern of a plane wave can be obtained from Equation (1), expressed in terms of the complex permittivity, permeability and conductivity of the medium [5].

$$
\nabla \times \mu_{r}^{-1}(\nabla \times E)-k_{0}^{2}\left(\varepsilon_{r}-\frac{j \sigma}{\omega \varepsilon_{0}}\right) E=0
$$

where $\mu_{r}$ is the relative permeability, $\boldsymbol{E}$ is the electric field vector, $k_{0}$ is the wavenumber in free space, $\varepsilon_{r}$ is the relative permittivity, $\omega$ is the angular frequency, $\sigma$ is conductivity and $\varepsilon_{0}$ is the permittivity of free space. Thus, the phasor is represented as $\vec{E}(r)=\vec{E}_{0} e^{-\gamma r}=\vec{E}_{0} e^{-\alpha r} e^{-j \beta r}$, where $r$ is the propagation distance, $\gamma\left(\mathrm{m}^{-1}\right)$ is the propagation constant, which is a complex quantity of the form $\gamma=\alpha+j \beta$, where the real part $\alpha(\mathrm{Np} / \mathrm{m})$ is the attenuation factor and the imaginary part $\beta(\mathrm{rad} / \mathrm{m})$ is the phase constant. Generally, oil reservoirs are a low-loss medium, that is $\omega \varepsilon \gg \sigma$. Hence, the irradiated EM waves are absorbed and attenuated as they propagate and spread within the medium [6,32]. Therefore, the attenuation factor $\alpha$ and phase constant $\beta$ becomes:

$$
\begin{aligned}
& \alpha=\frac{\sigma}{2} \sqrt{\frac{\mu}{\varepsilon}} \\
& \beta=\omega \sqrt{\mu \varepsilon}
\end{aligned}
$$

The skin depth/penetration depth $\delta$ in low-loss media is $\delta=\frac{2}{\sigma} \sqrt{\frac{\varepsilon}{\mu}}$, which is the inverse of the attenuation constant indicating the distance travelled by the wave in the medium before its amplitude reduces to $1 / \mathrm{e}$ of its initial value on the surface of the transmitter [5].

The half-wave dipole antenna was used in this study due to its extensive application in the EM enhanced oil recovery method, because of its radiation pattern $[17,28,29,33,34]$. This type of transmitter focuses the EM energy in the center of the dipole and exhibits an omnidirectional radiation pattern [6,32]. The relationship between the length of a half-wave dipole and its operating wavelength has been established by [6]. The conventional method is to design an antenna with a calculated length based on a predetermined frequency of propagation. However, considering the constraint and practical feasibility of transmitter placement in a reservoir well, it is reasonable to fix the antenna dimension based on reservoir geometry. Thereafter calculating its operating frequency over a range of possible values of reservoir EM properties $(\varepsilon, \mu$ and $\sigma)$ for optimal EM radiation. Hence, this research investigates the impact of reservoir effective EM properties at different fluid saturations on the operating efficiency of a half-wave dipole antenna. The ensuing EM propagation pattern of the antenna in a cylindrical oil reservoir model and its operating frequencies were analyzed. 


\section{Materials and Methods}

\subsection{Sample Preparation}

Berea sandstone samples with a porosity of 0.21 and permeability $230 \mathrm{mD}(7.5 \mathrm{~cm}$ long and $3.8 \mathrm{~cm}$ in diameter) were prepared for saturation and EM properties were measurement. Crude oil of density $0.802 \mathrm{~g} / \mathrm{cm}^{3}$ and viscosity $7.5 \mathrm{cp}$ obtained from Angsi field was used in this study, while magnetite nanoparticles were purchased from Sigma-Aldrich for nanofluid preparation. An 11,000 ppm brine solution was prepared through the dissolution of $0.11 \mathrm{~g}$ of $\mathrm{NaCl}$ in $100 \mathrm{~mL}$ of distilled water. $\mathrm{Fe}_{3} \mathrm{O}_{4}$ nanofluid $\left(0.05 \mathrm{wt} \%\right.$ ) was subsequently prepared by dispersing $0.05 \mathrm{~g}$ of $\mathrm{Fe}_{3} \mathrm{O}_{4}$ in $100 \mathrm{~mL}$ of the earlier prepared brine solution and sonicated for $30 \mathrm{~min}$ to achieve homogenous dispersion. The sandstone samples were saturated with the respective formation fluid (brine, crude oil and nanofluid) in a pressurized $(1.85 \mathrm{MPa})$ stainless-steel autoclave placed in an oven at $120^{\circ} \mathrm{C}$ for $48 \mathrm{~h}$ for effective saturation (Figure 1). The shape and size distribution of the dry and saturated sandstone grain were examined with the aid of a field emission scanning electron microscope (FESEM) operated at $5 \mathrm{kV}$. The analysis was conducted using a FESEM (SUPRA 55VP Carl Zeiss AG, Germany) equipped with an energy dispersive spectrum (EDS) analyzer, which gives the elemental distribution to confirm the inclusion of the saturating fluid (brine, crude oil and nanofluid) in the rock sample.

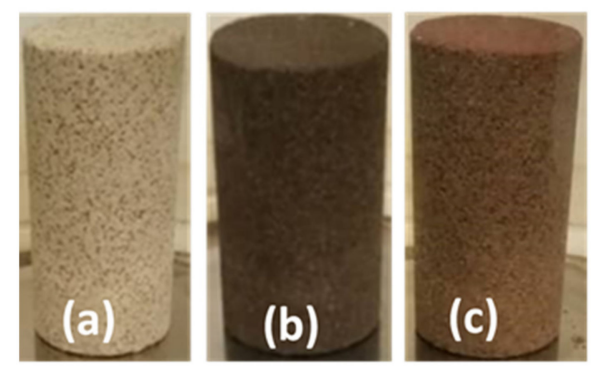

Figure 1. Berea sandstone samples (a) dry sample, (b) sample saturated with crude oil and (c) sample saturated with crude oil and magnetite nanofluid.

\subsection{Reservoir Rock Sample Electromagnetic Properties}

The magnetic property of the sandstone samples was characterized using a vibrating sample magnetometer (VSM GMW 7400, Lakeshore, Westerville, OH, USA). For the magnetic response measurement, the VSM experiment was conducted applying a maximum magnetic field of $1500 \mathrm{G}$ on the magnetite nanoparticle and sandstone samples. The hysteresis loop, magnetization, coercivity and retentivity of the samples were obtained. The dielectric permittivity of the samples was measured within a frequency range of $1 \mathrm{MHz}$ to $4 \mathrm{GHz}$ using a vector network analyzer (ENA E5071C). The frequency-dependent conductivity $\sigma_{\mathrm{ac}}(\mathrm{S} / \mathrm{m})$ was computed from the measured complex relative permittivity value using Equation (4).

$$
\sigma_{\mathrm{ac}}=2 \pi f \varepsilon^{\prime \prime} \varepsilon_{0}
$$

where $\mathrm{f}$ is the frequency, $\varepsilon^{\prime \prime}$ is the imaginary part of permittivity and $\varepsilon_{0}$ is the permittivity of free space.

\subsection{Reservoir Model Geometry and Electromagnetic Propagation Simulation}

EM propagation of a 17-mm long half-wave dipole antenna in a reservoir environment was simulated based on the earlier measured EM properties $(\varepsilon, \sigma$ and $\mu$ ) using COMSOL Multiphysics software. Figure 2 shows the schematic representation of the conceptual geometrical model deployed in the simulation study, where the transmitter was positioned at the injection well. 


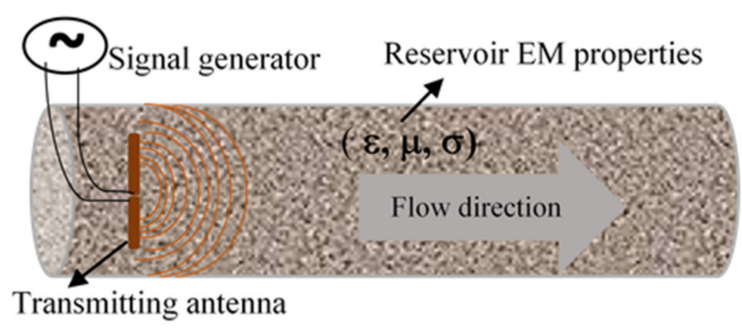

Figure 2. Schematic diagram of reservoir geometry with electromagnetic (EM) simulation.

A user-controlled mesh option was utilized for the numerical discretization to create a mesh sequence with highly refined elements on the antenna structure and at the edges of the geometry (as shown in Figure 3). Since this is an electromagnetic problem, the maximum mesh element size $S_{\max }$ follows the Nyquist criterion $S_{\max }<\frac{c}{2 f \sqrt{\varepsilon^{\prime} \mu^{\prime}}}$ [35]. A combination of tetrahedral and rectangular mesh elements was used, and the mesh was divided into three sequences, the first of which, representing the sandstone region, has a maximum element size and growth rate of $1.342 \mathrm{~cm}$ and 1.5 , respectively. The second mesh sequence used for the antenna structure has a $S_{\max }$ of $0.01 \mathrm{~cm}$, a maximum element growth rate of 1.5 and a curvature factor of 0.6. The maximum element size of the third sequence (the lumped port) is $1.25 \times 10^{-3} \mathrm{~cm}$.

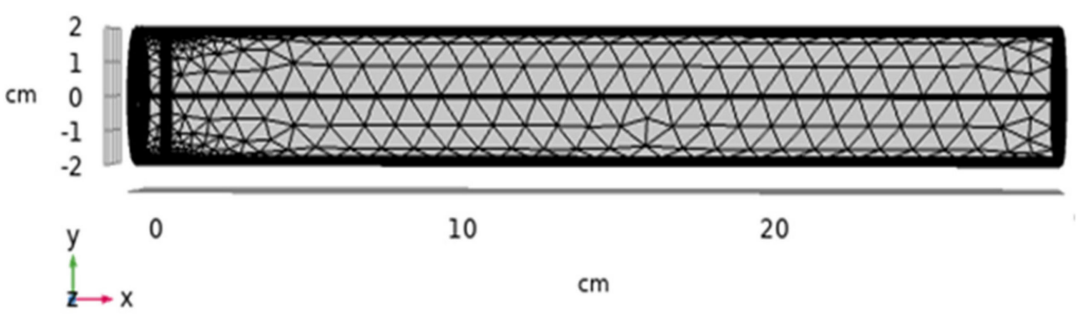

Figure 3. Antenna and sandstone core geometry mesh.

The perfectly matched layer (PML) boundary condition was used to eliminate the boundary reflection phenomenon for computational convenience. The PML is a domain with an anisotropic and complex-valued permittivity and permeability used for encapsulating a geometric domain of interest for the frequency-domain solution of Maxwell's equations. Our choice of a polynomial stretched-coordinate perfectly matched layer (SC-PML) boundary condition that absorbs omnidirectionally was inspired by the report of [36]. The details of the geometric dimensions used for building the model depicted in Figure 3 are given in Table 1. Wave excitation was produced by applying voltage difference at the lumped port (the gap between the two antenna arm lengths) with a coaxial cable transmission line with a characteristic impedance $Z_{L}$ of $50 \Omega$. The electric field distribution at different distances from the antenna ensuing from the EM radiation was obtained by solving the wave equation in lossy media [37]. Accordingly, the three-dimensional far-field distribution was obtained.

Table 1. Geometric model parameters.

\begin{tabular}{cc}
\hline Parameter & Value \\
\hline Reservoir model length $(\mathrm{cm})$ & 30.5 \\
Reservoir model diameter $(\mathrm{cm})$ & 4.0 \\
Transmitter distance $(\mathrm{cm})$ & 1.0 \\
Antenna arm length $(\mathrm{mm})$ & 17.0 \\
Antenna radius $(\mathrm{mm})$ & 1.0 \\
Gap $(\mathrm{mm})$ & 1.0 \\
\hline
\end{tabular}




\section{Results and Discussion}

\section{1. $\mathrm{Fe}_{3} \mathrm{O}_{4}$ Nanofluid Characterization}

The FESEM image of the $\mathrm{Fe}_{3} \mathrm{O}_{4}$ nanoparticle used to prepare the magnetite nanofluid for saturating the core rock sample is shown in Figure 4a. The FESEM image shows that the nanoparticles have a spherical shape and an average particle diameter of $36 \mathrm{~nm}$. The spherical shape of the nanoparticles combined with its small particle size is beneficial for enhancing oil recovery [38,39]. The nanoparticles will thus possess high mobility through pore throats and good adsorption of oil molecules and will easily detach oil molecules from the rock surface leading to an improved recovery factor. In contrast, the large particle size in the order of microns could solidify upon application of an external magnetic field and cause pore throat blockage [40]. Figure $4 \mathrm{~b}$ displays the hysteresis curve of the magnetite nanoparticle, which has a saturation magnetization (Ms) of $42.3 \mathrm{emu} / \mathrm{g}$ and retentivity (Mr) of $3.0134 \mathrm{emu} / \mathrm{g}$. The prepared $\mathrm{Fe}_{3} \mathrm{O}_{4}$ nanofluid has a DC conductivity of $2.19 \mathrm{~S} / \mathrm{m}$ and dielectric permittivity of 74.2 at $600 \mathrm{MHz}$.
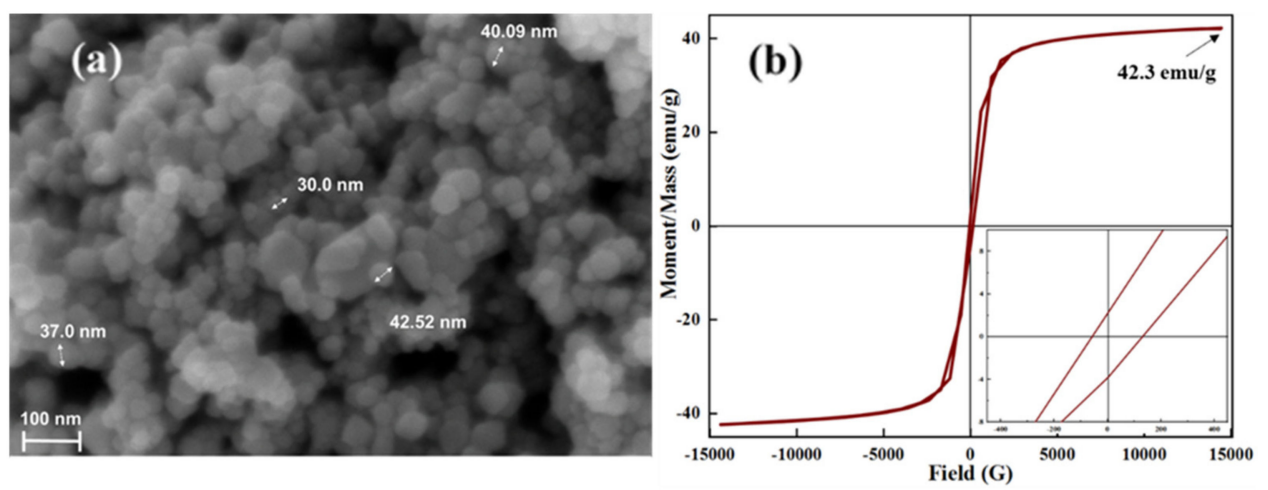

Figure 4. (a) Field emission scanning electron microscope (FESEM) image showing particle size and the shape of a magnetite nanoparticle and (b) hysteresis loop, the inset shows a magnified image of the initial magnetization.

\subsection{Reservoir Rock Sample Characterization}

A combination of a FESEM micrograph and an EDS elemental analysis of the Berea sandstone sample is presented in Figure 5. The dry sandstone grain and pore distribution are shown in Figure 5a, which indicates that the sandstone sample is dominated with macropores with a pore diameter of the order of $100 \mu \mathrm{m}$. This implies that the magnetite nanoparticles will possess ample mobility during core flooding without causing pore throat blockage, as the particle size is less than $50 \mathrm{~nm}$. Moreover, it has been reported that the frequency dependence of $\varepsilon^{\prime}$ of saturated sandstone greatly depends on pore space surface area to volume ratio [41].

The elemental distribution of the pore space is indicated in Figure $5 b$ by the EDS spectrum, which is comprised largely of silicon (31.43 wt $\%$ ) of sandstone silica and oxygen $(50.39 \mathrm{wt} \%)$ of connate water and carbon $(18.18 \mathrm{wt} \%)$. Hence, a combination of the pore structure and elemental composition are important background knowledge, which can help determine the influence of the injected fluid content on EM wave propagation within the medium, as will be shown in subsequent sections. The presence of the injected crude oil and saturating nanofluid is confirmed in Figure $5 d$, which shows the EDS element distribution analysis within a pore space (Figure $5 c$ ) of the sandstone sample. A combination of the high carbon $(C)$ content in the spectrum confirms the presence of the saturating crude oil, which is $61.16 \mathrm{wt} \%$ of the pore content. The presence of magnetite nanofluid is likewise confirmed by the Fe peak in the EDS spectrum and is relatively sparse $(0.92 \mathrm{wt} \%)$ due to the small amount $(0.05 \mathrm{wt} \%)$ of $\mathrm{Fe}_{3} \mathrm{O}_{4}$ nanoparticles dispersed in the $\mathrm{NaCl}$ used to prepare the nanofluid. This small concentration of nanoparticles was used to avoid nanoparticle retention within the porous structure, as this has been earlier reported [42]. 

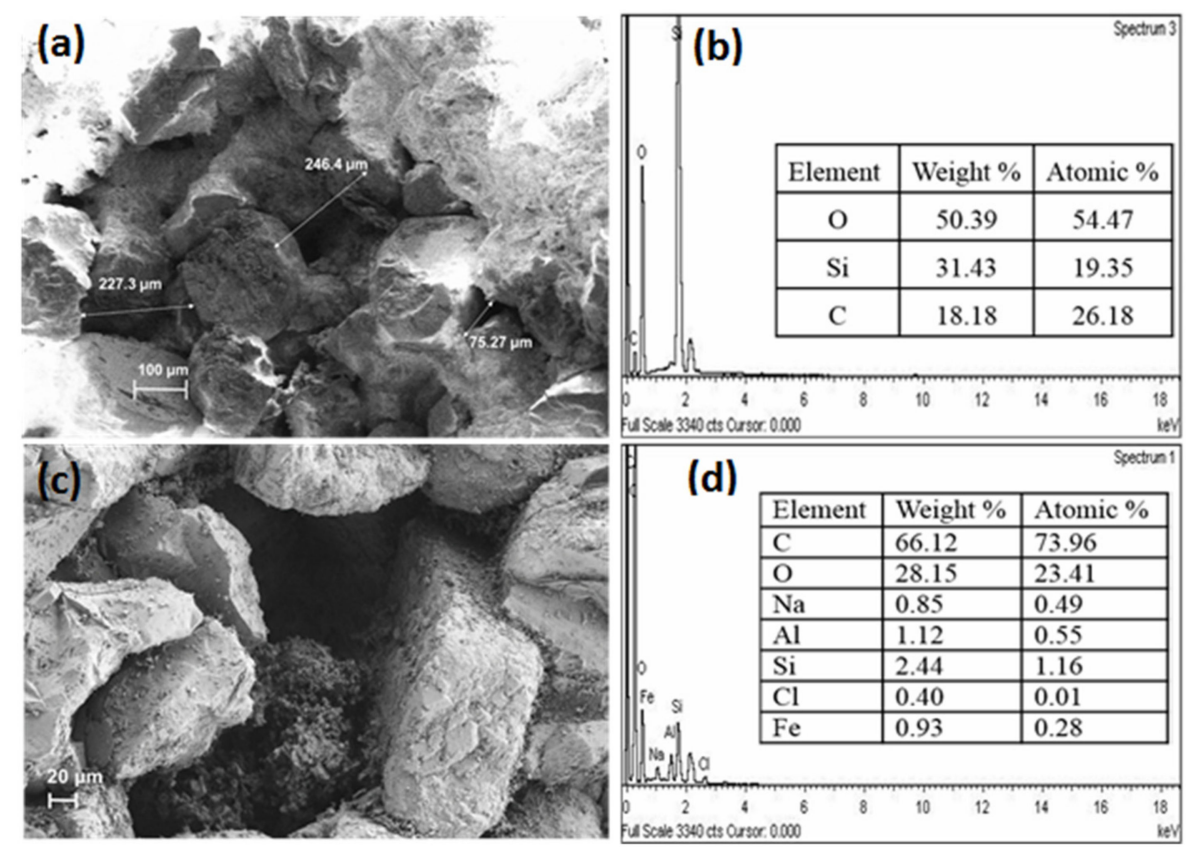

Figure 5. FESEM results showing (a) pore size distribution of the dry sandstone sample, (b) EDS analysis of element distribution of dry sandstone, (c) an image of a pore throat mapped after saturating with oil, brine and $\mathrm{Fe}_{3} \mathrm{O}_{4}$ nanofluid and (d) EDS analysis of elemental distribution confirming the presence of the injected oil and nanofluid.

\subsection{Reservoir Rock Electromagnetic Properties}

Figure 6a shows the variation of relative dielectric permittivity $\varepsilon^{\prime}$ of the sandstone sample under the different possible scenarios encountered in nanofluid core flooding experiments. It is apparent from the figure that dielectric dispersion generally dominates the lower frequency portion (from $1 \mathrm{MHz}$ to $1.5 \mathrm{GHz}$ ) of our measurement range, after which the frequency dependence of $\varepsilon^{\prime}$ becomes weaker and attains quasi-stable values. Since relative permittivity is a measure of the degree of polarization occurring in a material [43], the various exhibited peaks at different frequencies could be attributed to specific polarization mechanisms [41], of which dipolar mechanisms are dominant in this case. It is noteworthy to point out that the $\varepsilon^{\prime}$ of the sample not only increases with the inclusion of brine and nanofluid, but dispersion peaks also shift to a lower frequency.
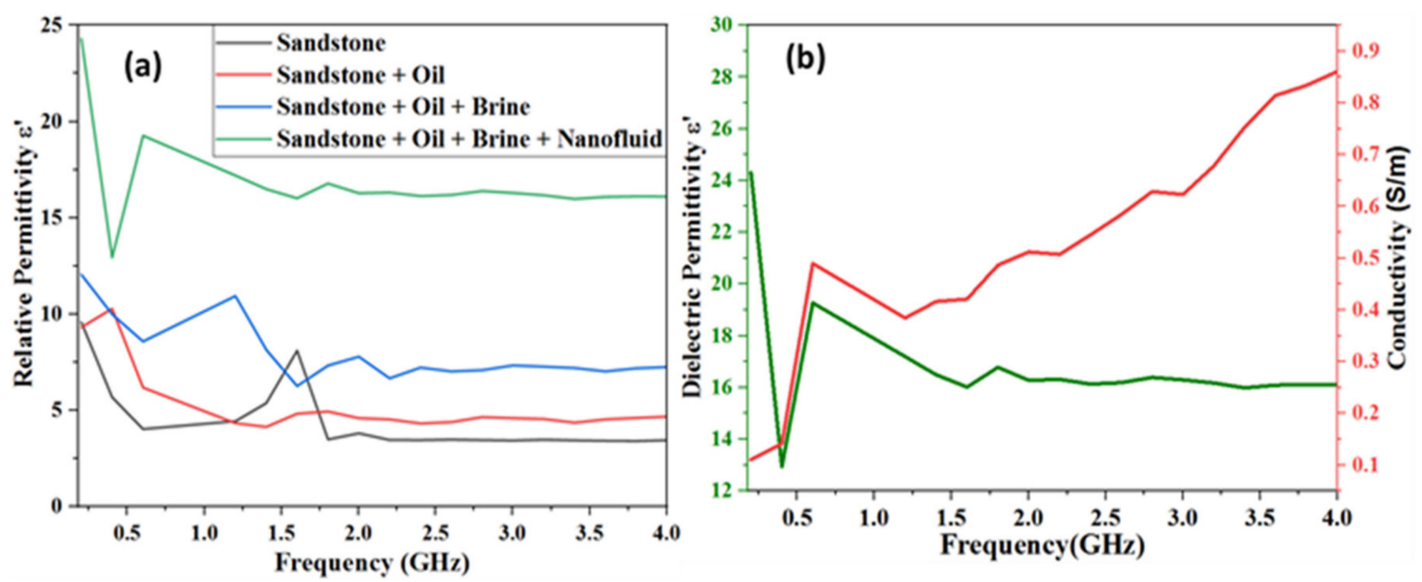

Figure 6. (a) Dielectric permittivity of the sandstone sample at different saturations and (b) permittivity and conductivity variation of the sample saturated with brine, oil and nanofluid. 
The frequency-dependent conductivity $\sigma_{\mathrm{ac}}$ (obtained from the imaginary permittivity according to Equation (4)) and $\varepsilon^{\prime}$ of the sandstone sample saturated with $40 \%$ oil, $60 \%$ brine and nanofluid is shown in Figure $6 \mathrm{~b}$. The frequency-dependent conductivity of the medium displays an increasing trend with frequency as opposed to the decreasing value of $\varepsilon^{\prime}$. Moreover, the effective conductivity $\sigma_{\text {eff }}$ of the reservoir medium is composed of the ohmic $\sigma_{\text {ohmic }}$, and the dielectric loss part $\sigma_{\text {ac }}$ (the frequency-dependent) as $\sigma_{\text {eff }}=\sigma_{\text {ohmic }}+\sigma_{a c}$. We therefore conclude from these results that the irradiated EM wave should be varied within a frequency range less than $2 \mathrm{GHz}$. This will enable effective propagation of the EM wave, as attenuation of the EM wave will increase at a higher frequency (as indicated by Equation (2)) leading to a reduction of the penetration depth $\delta$ due to increasing conductivity at a higher frequency, where $\varepsilon^{\prime}$ is almost constant. Figure $6 \mathrm{a}, \mathrm{b}$ clearly indicates that approximately $2 \mathrm{GHz}$ is the upper bound of frequency for an effective response of the reservoir materials to the irradiated EM wave within the tested samples. This is in agreement with the report of Fanchi [44], who placed the lower frequency limit at $300 \mathrm{MHz}$ and the upper bound at 1-2 GHz for effective EM propagation in the reservoir medium. Therefore, our subsequent investigation in this work was restricted to the frequency limit of $100 \mathrm{MHz}$ to $2 \mathrm{GHz}$.

It can be concluded from the results that, while the injection of $\mathrm{Fe}_{3} \mathrm{O}_{4}$ nanofluid increased the overall EM response of the reservoir, another important point is the reduction of the required EM frequency for the EM-assisted nanoflooding experiment, hence increasing the EM penetration depth, polarization effect and improved activation of the injected nanofluid for stimulation of the residual oil. The sandstone sample shows a weak magnetic property (Ms of $10.361 \mathrm{memu} / \mathrm{g}$ ), which conforms with earlier reports in the literature that most reservoir rocks do not possess intrinsic magnetic properties and are therefore described electrically by the conductive and capacitive properties [45]. However, a variation of the saturation magnetization could be noticed with different fluid contents. Thus, the EM response of the medium entirely depends on the volume fraction of the injected nanofluid. Table 2 summarizes the measured and calculated EM parameters of the sandstone samples that were considered for the simulation study.

Table 2. Electromagnetic properties of sandstone samples at different fluid saturations.

\begin{tabular}{cccccc}
\hline Formation & $\begin{array}{c}\text { Dielectric } \\
\text { Permittivity }\end{array}$ & $\begin{array}{c}\text { Conductivity } \\
\left(\times \mathbf{1 0}^{-\mathbf{2}} \mathbf{S} / \mathbf{m}\right)\end{array}$ & $\begin{array}{c}\text { Magnetization } \\
\left(\times \mathbf{1 0}^{-\mathbf{3}} \mathbf{~} \mathbf{m u} / \mathbf{g}\right)\end{array}$ & Coercivity (G) & $\begin{array}{c}\text { Retentivity } \\
\left(\times \mathbf{1 0}^{-\mathbf{9}} \mathbf{~} \mathbf{~ m u} / \mathbf{g}\right)\end{array}$ \\
\hline Dry Sandstone & 4.0 & 3.276 & 10.361 & 47.82 & 91.89 \\
Sandstone + Oil & 6.1 & 2.396 & 4.367 & 37.59 & 29.43 \\
Sandstone + Oil + Brine & 8.1 & 3.487 & 6.221 & 23.91 & 32.05 \\
$\begin{array}{c}\text { Sandstone + Oil + Brine } \\
\text { + Nanofluid }\left(\mathrm{Fe}_{3} \mathrm{O}_{4}\right)\end{array}$ & 19.1 & 7.732 & 10.601 & 31.66 & 24.30 \\
\hline
\end{tabular}

\subsection{EM Wave Propagation}

\subsubsection{Antenna Impedance and Return Loss}

The simulated input impedance at the lumped port of the antenna is presented in Figure 7 for different operating frequencies ranging from $100 \mathrm{MHz}$ to $2.0 \mathrm{GHz}$. For an antenna to propagate and deliver maximum power, the input impedance is expected to match with the characteristic impedance of the transmission line according to $Z_{L}=Z_{A}$, where $Z_{L}=R_{L}+j X_{L}$ is the transmission line characteristic impedance (composed entirely of reactance (real) part $R_{\mathrm{L}}=50 \Omega$ and capacitance (imaginary) part $\left.X_{\mathrm{L}}=0\right)$ and $Z_{A}=R_{A}+j X_{A}$ is the antenna input impedance. Therefore, the simulated antenna will resonate (propagate maximum power) at a frequency where the imaginary impedance is closest to zero, with a corresponding real impedance close to $50 \Omega$. It was observed that the real part of the input impedance of the antenna approaches $50 \Omega$ at $0.2 \mathrm{GHz}(56.54 \Omega), 1.4 \mathrm{GHz}(51.22 \Omega), 1.5 \mathrm{GHz}$ $(56.01 \Omega), 1.7 \mathrm{GHz}(51.39 \Omega)$ and $1.8 \mathrm{GHz}(42.62 \Omega)$. Nonetheless, the antenna cannot resonate at these frequencies due to the large respective imaginary parts, $111.79 \Omega, 10.82 \Omega, 16.33 \Omega$ and $35.85 \Omega$. The real and imaginary parts of the antenna input impedance in sandstone saturated with oil, brine and 
nanofluid are presented in Figure $7 \mathrm{c}$, as this is of interest for the core flooding process. The imaginary part is noticed to approach zero at $0.8 \mathrm{GHz}(3.82 \Omega)$ and $1.3 \mathrm{GHz}(8.96 \Omega)$; this indicates that power stored in the near field (non-radiated power) is minimal at these frequencies. Likewise, the real part of the impedance at these frequencies are $40.51 \Omega$ and $46.35 \Omega$, respectively; hence the antenna propagates the maximum input power at $800 \mathrm{MHz}$ and $1.3 \mathrm{GHz}$.
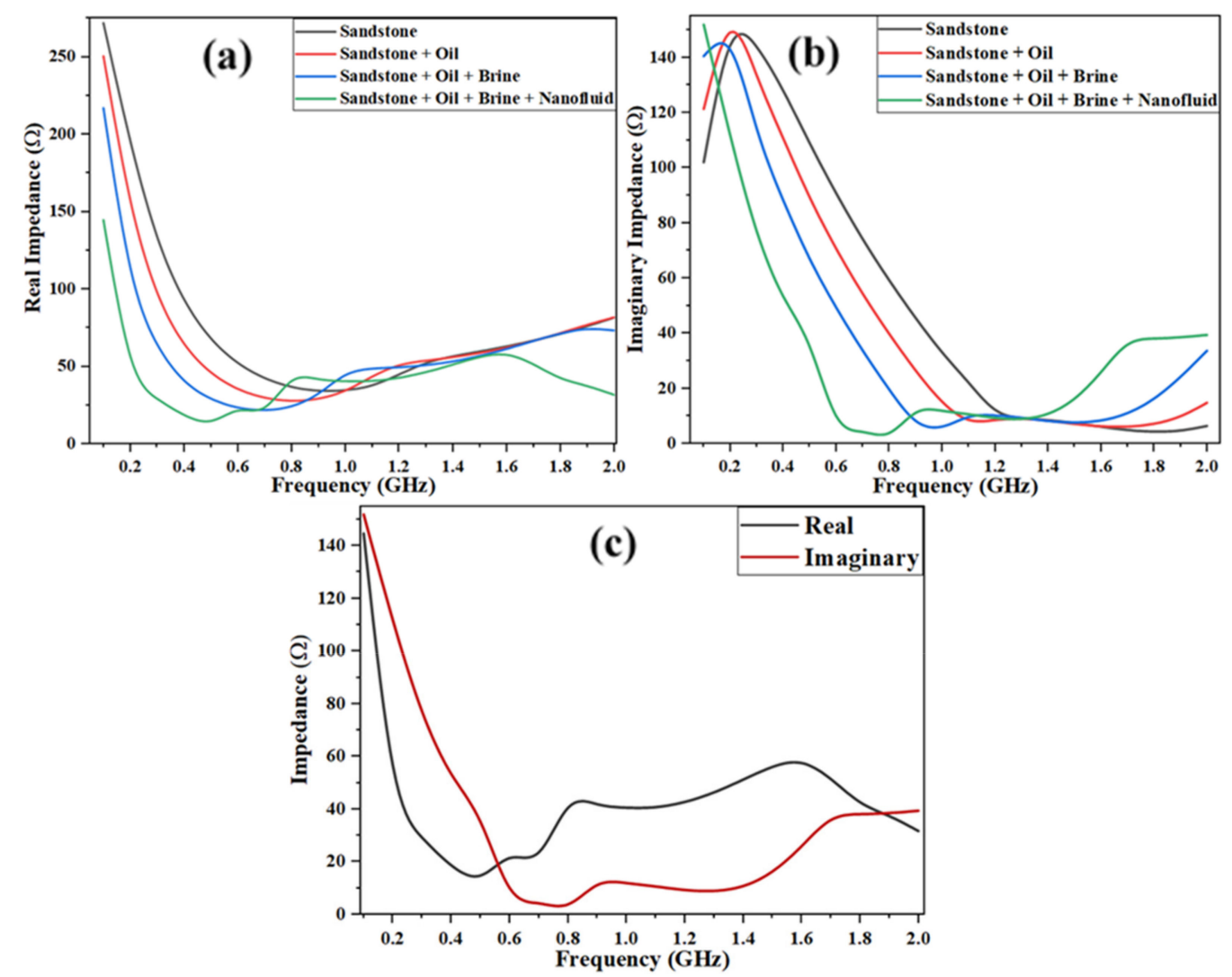

Figure 7. Input impedance of the antenna in sandstone medium at different saturations; (a) real impedance, (b) the imaginary part and (c) real and imaginary impedance in sandstone containing oil, brine and nanofluid.

Figure 8 shows the simulated return loss of the antenna in four different media, dry sandstone, sandstone saturated with oil, sandstone saturated with oil and brine and sandstone saturated with oil, brine and nanofluid. The essence of analyzing the reflection loss peaks is to determine frequencies at which the least amount of input signal is reflected by the antenna in order to maximize energy delivery to reservoir materials for stimulation and recovery of trapped oil. The antenna has return loss $<-10 \mathrm{~dB}$ in the range; between $1.09 \mathrm{GHz}$ and extends over $2 \mathrm{GHz}(0.91 \mathrm{GHz}$ bandwidth), between $990 \mathrm{MHz}$ and $2 \mathrm{GHz}$ (1.01 GHz bandwidth), between $900 \mathrm{MHz}$ and $2 \mathrm{GHz}$ (1.1 GHz bandwidth), and between $580 \mathrm{MHz}$ and $1.68 \mathrm{GHz}(1.1 \mathrm{GHz}$ bandwidth) within the aforementioned four scenarios, respectively. The curve indicates that the antenna has a single reflection loss peak with a corresponding value of $-20.708 \mathrm{~dB}$ at $1.3 \mathrm{GHz}$ and $-20.575 \mathrm{~dB}$ at $1.2 \mathrm{GHz}$ for the dry sample and when saturated with oil, respectively. However, the antenna exhibits double reflection loss peaks in the sandstone containing oil and brine $(-21.12 \mathrm{~dB}$ at $1.0 \mathrm{GHz}$ and $-21.37 \mathrm{~dB}$ at $1.4 \mathrm{GHz})$ and that containing oil, brine and magnetite nanofluid $(-18.91 \mathrm{~dB}$ at $800 \mathrm{MHz}$ and $-20.00 \mathrm{~dB}$ at $1.3 \mathrm{GHz})$. The double reflection loss peaks of the antenna within these two media could be attributed to the electrical length of the antenna. The antenna approaches the limit of an electrically large antenna at the high-frequency peak (shorter wavelength of the propagated EM wave), and an electrically small antenna at the lower frequency peak corresponding to the longer propagating wavelength $[46,47]$. 


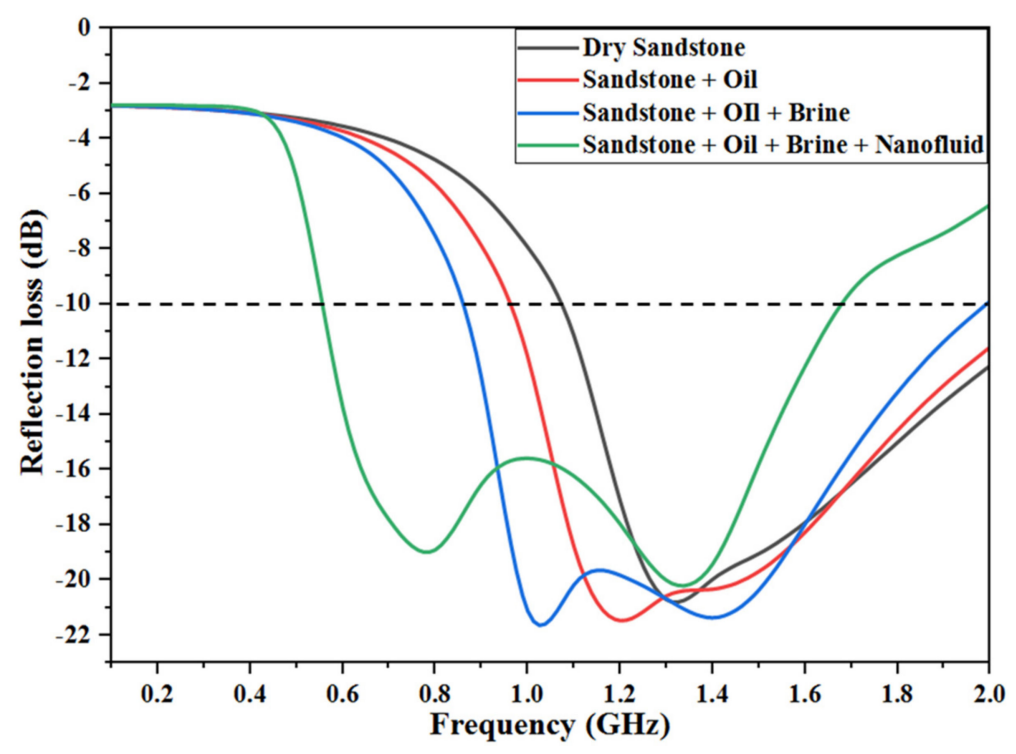

Figure 8. Antenna return loss $(\mathrm{dB})$ at different operating frequencies in different formation fluid saturations.

It can be inferred from the return loss graph that an increasing value of the permittivity of the medium due to an increase in the injected polar molecules causes the reflection loss peaks (equivalent to antenna resonant frequencies) to shift to lower frequencies. This trend is most prominent after including magnetite in the saturating fluid, with one of its reflection losses peaks $(-18.91 \mathrm{~dB})$ occurring at $800 \mathrm{MHz}$. This is a particularly important finding in the application of EM waves for the activation of injected nanoparticles, as it indicates that approximately $95 \%$ of the EM power supplied to the antenna is propagated within the medium.

As earlier mentioned, the resonant frequency of the Berea sandstone sample saturated with oil, brine and magnetite nanofluid was observed to be $600 \mathrm{MHz}$, implying that there was a maximum response to the irradiated EM wave [48]. This connotes that the maximum response to externally applied EM waves will be obtained by tuning the EM frequency close to this value (600 MHz). Hence, a reasonable compromise could be made between applying EM waves at the resonant frequency of the transmitting antenna $(800 \mathrm{MHz})$ or that of the reservoir medium $(600 \mathrm{MHz})$. Albeit, it could be preferable to apply EM waves of frequency $600 \mathrm{MHz}$ due to two compelling reasons. Firstly, the ability of the antenna to absorb and radiate roughly $94 \%$ of the input power (as indicated by the reflection loss of $-13.75 \mathrm{~dB}$ ) [48]. Secondly, maximum energy could be transferred to reservoir materials at this frequency due to resonance. Subsequently, sufficient EM energy is available for polarizing the magnetite nanoparticles and also overcoming the isotropic Van der Waal's attractive forces between the nanoparticles. This is brought about through reduction of Van der Waal interaction free energy relating to the Hamaker constant and anisotropic dipolar forces between the magnetite nanoparticles $[49,50]$. Thus, improved activation of the nanoparticles is achieved. Table 3 presents a summary of the antenna characteristics at different fluid saturations. The return loss, input impedance, bandwidth and by implication the antenna radiation efficiency is significantly affected by the fluid content of the medium.

Table 3. Antenna characteristics at different saturations.

\begin{tabular}{ccccc}
\hline Parameters & Dry Sandstone & Oil Saturated & Oil and Brine Saturated & Oil, Brine and Nanofluid Saturated \\
\hline Resonant Frequency $(\mathrm{GHz})$ & 1.30 & 1.20 & 1.00 and 1.40 & 0.80 and 1.30 \\
Return Loss $(\mathrm{dB})$ & -20.70 & -21.47 & -21.12 and -21.36 & -18.911 and -20.00 \\
Real Impedance $(\Omega)$ & 52.04 & 50.62 & 44.39 and 53.31 & 40.51 and 46.35 \\
Imaginary Impedance $(\Omega)$ & 9.22 & 8.50 & 6.13 and 8.21 & 3.82 and 8.96 \\
Bandwidth $(\mathrm{GHz})$ & 0.91 & 1.01 & 1.10 & 1.10 \\
\hline
\end{tabular}




\subsubsection{Transmitter Propagation Pattern}

Figure 9 shows the simulated radiating pattern of the antenna. It can be seen from Figure $9 a, b$ that the antenna becomes directional and beamforming with increasing frequencies (beyond $700 \mathrm{MHz}$ ) as indicated by the gradual appearance of side lobes. As a result, more propagation distance was covered in one direction. However, the angular coverage decreases, and the radiation pattern is non-uniform, implying an unequal distribution of the radiated energy. Therefore, the antenna should be operated at a frequency less than $800 \mathrm{MHz}$ to achieve a uniform radiating field pattern, which is one of the major goals of downhole antenna design [28]. The radiation pattern was found to be uniform at $600 \mathrm{MHz}$, as shown by the omnidirectional pattern in Figure $9 \mathrm{~b}$, indicating that a roughly equal amount of power was radiated from all sides of the antenna. Interestingly, this coincides with the resonant frequency of the medium as earlier determined. Figure $9 \mathrm{c}$ shows a three-dimensional image of the far-field propagation pattern obtained at $600 \mathrm{MHz}$, indicating the E-field distribution around the antenna within the sandstone medium saturated with oil, brine and nanofluid.

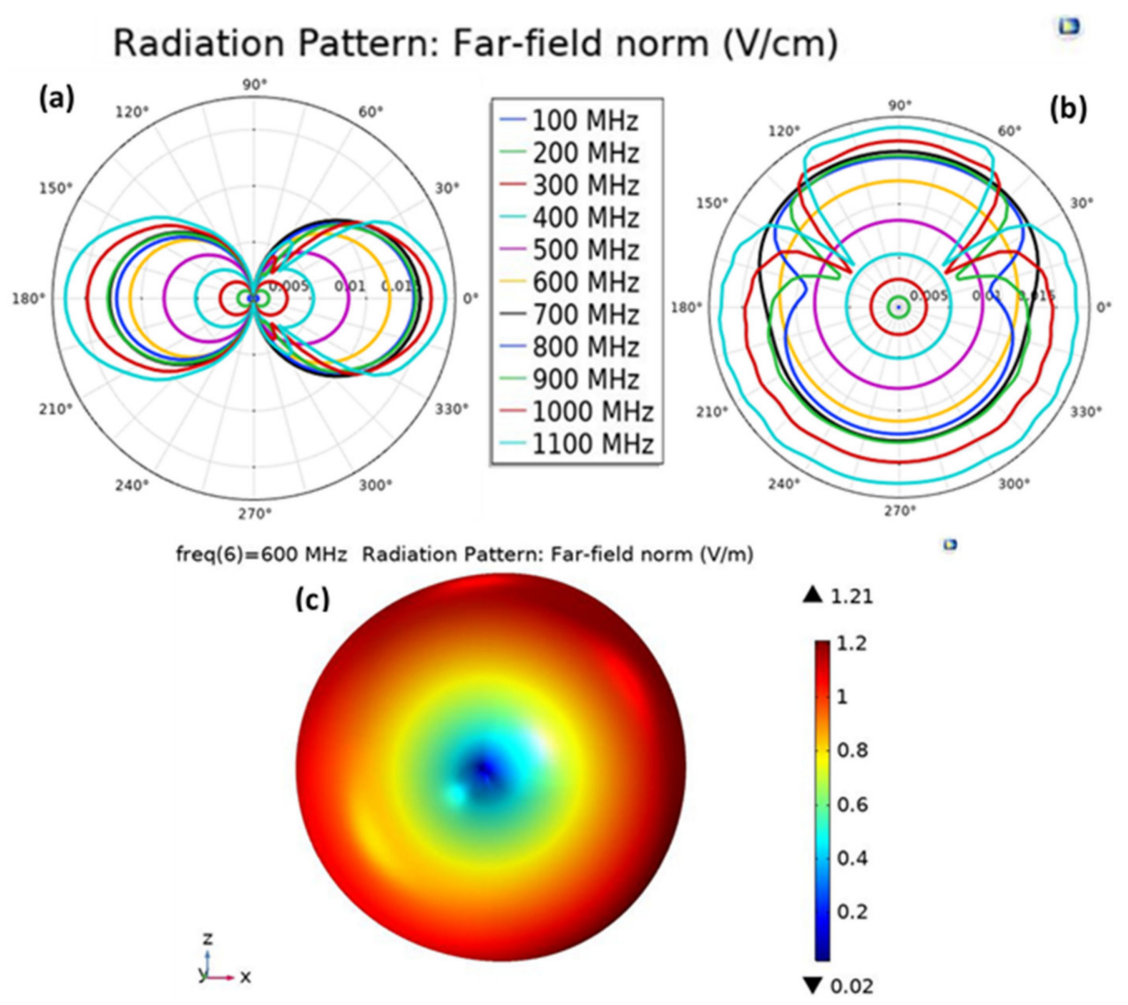

Figure 9. A far-field distribution pattern in sandstone saturated with oil, brine and nanofluid showing (a) a polar plot of the side view from the XZ plane (conical cut at theta), (b) a polar plot of the top view from the XY plane (planar cut at phi) and (c) a 3D polar plot at $600 \mathrm{MHz}$

It is important to establish that the far-field pattern, antenna gain, radiated power (Figure 10) and other parameters are evaluated and quantified within this region [32].

Figure 11a shows the antenna propagation within the modelled reservoir geometry. The figure gives a visual representation of E-field distribution within the medium. The EM wave attenuation phenomenon is likewise visible from the figure, where the field strength is maximum at the antenna surface, and gradually diminishing at a distance. The penetration depth at $600 \mathrm{MHz}$ was computed as $5.7 \mathrm{~cm}$. An alternate setup for increasing the EM propagation distance by using double transmitter placement is shown in Figure 11b. 

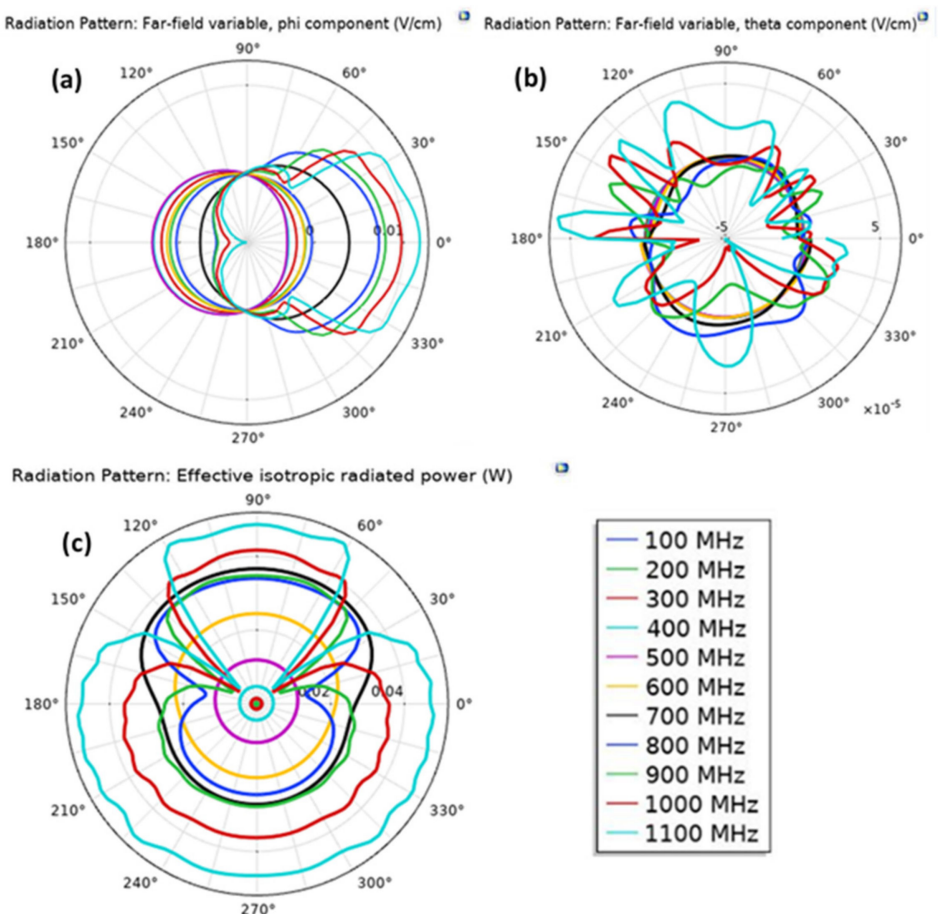

Figure 10. A two-dimensional polar plot of the far-field pattern showing (a) a radiation pattern along the phi component, (b) a radiation pattern along the theta component and (c) the distribution of the radiated isotropic power around the antenna.
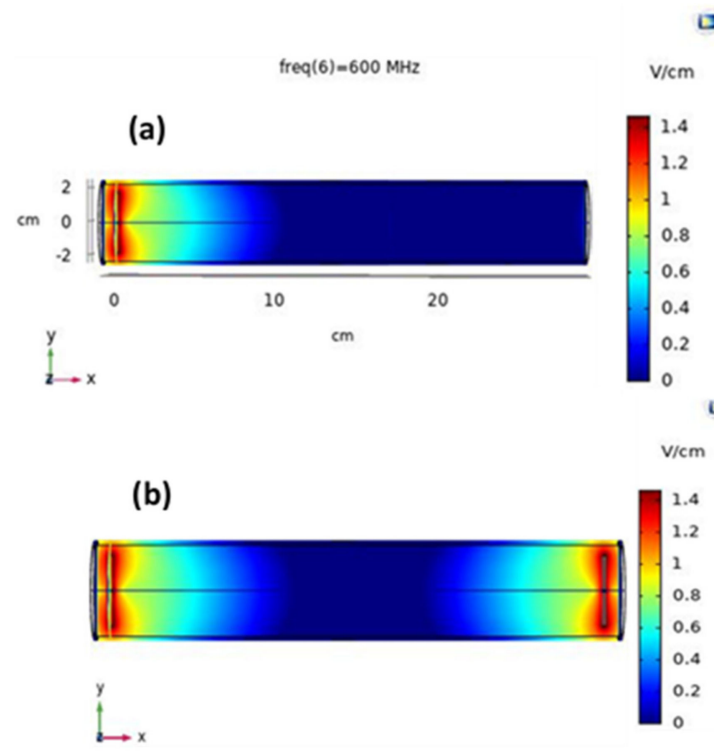

Figure 11. (a) The E-field distribution of the antenna in sandstone saturated with oil, brine and nanofluid at $600 \mathrm{MHz}$ and (b) the E-field distribution using a double antenna, with the transmitter placed in the injection and production well.

\section{Conclusions}

The EM propagation of a half-wave dipole antenna in a cylindrical reservoir model has been evaluated. The far-field patterns of the geometry are illustrated using 2D and 3D diagrams to depict the propagation at different operating frequencies. The antenna was observed to exhibit double reflection loss peaks in the sandstone containing oil and brine $(-21.12 \mathrm{~dB}$ at $1 \mathrm{GHz}$ and $-21.37 \mathrm{~dB}$ at $1.4 \mathrm{GHz})$, and that containing oil, brine and magnetite nanofluid $(-18.91 \mathrm{~dB}$ at $800 \mathrm{MHz}$ and $-20.00 \mathrm{~dB}$ at $1.3 \mathrm{GHz})$. Evaluation of the radiating far-field pattern reveals that the E-field distribution, isotopic radiated power 
and phi and theta components become directional with the appearance of side lobes as the frequency increases beyond $700 \mathrm{MHz}$. Hence, operating the antenna at $600 \mathrm{MHz}$ could improve the efficiency of EM propagation and energy transfer within the medium, as this coincides with the resonant frequency of the reservoir medium.

Author Contributions: Conceptualization, N.Y.; Funding acquisition, N.Y.; Investigation, F.A.W.; Methodology, F.A.W. and A.R.; Software, H.S. and A.R.; Visualization, F.A.W.; Supervision, N.Y. and A.S.; Project Administration, M.G., Writing—original draft, F.A.W.; Formal analysis, F.A.W., Writing—review and editing, N.Y., A.S., H.S., A.R. and M.G.

Funding: This research received no external funding.

Acknowledgments: The authors express their appreciation to Universiti Teknologi PETRONAS for providing excellent research facilities and financial support through the graduate assistantship scheme.

Conflicts of Interest: The authors declare no conflict of interest.

\section{References}

1. Ghazanfari, E.; Pervizpour, M.; Pamukcu, S. Mathematical Modeling of Electrically Assisted Hydrocarbon Transport in Porous Media. In Proceedings of the GeoCongress, Oakland, CA, USA, 25-29 March 2012; pp. 2302-2311.

2. Ryoo, S.; Rahmani, A.R.; Yoon, K.Y.; Prodanović, M.; Kotsmar, C.; Milner, T.E.; Johnston, K.P.; Bryant, S.L.; Huh, C. Theoretical and experimental investigation of the motion of multiphase fluids containing paramagnetic nanoparticles in porous media. J. Petrol. Sci. Eng. 2012, 81, 129-144. [CrossRef]

3. Prigiobbe, V.; Ko, S.; Huh, C.; Bryant, S.L. Measuring and modeling the magnetic settling of superparamagnetic nanoparticle dispersions. J. Colloid Interface Sci. 2015, 447, 58-67. [CrossRef] [PubMed]

4. Abdulrahman, M.M.; Meribout, M. Antenna array design for enhanced oil recovery under oil reservoir constraints with experimental validation. Energy 2014, 66, 868-880. [CrossRef]

5. Guru, B.S.; Hiziroglu, H.R. Electromagnetic Field Theory Fundamentals; Cambridge University Press: Cambridge, UK, 2009.

6. Balanis, C.A. Modern Antenna Handbook; John Wiley \& Sons: Hoboken, NJ, USA, 2011.

7. Revil, A. Effective conductivity and permittivity of unsaturated porous materials in the frequency range 1 mHz-1 GHz. Water Resour. Res. 2013, 49, 306-327. [CrossRef] [PubMed]

8. Irfan, S.A.; Shafie, A.; Yahya, N.; Zainuddin, N. Mathematical Modeling and Simulation of NanoparticleAssisted Enhanced Oil Recovery-A Review. Energies 2019, 12, 1575. [CrossRef]

9. Adebayo, L.L.; Soleimani, H.; Yahya, N.; Abbas, Z.; Ridwan, A.T.; Wahaab, F.A. Investigation of the Broadband Microwave Absorption of Citric Acid Coated Fe3O4/PVDF Composite Using Finite Element Method. Appl. Sci. 2019, 18. [CrossRef]

10. Kovaleva, L.; Davletbaev, A.; Babadagli, T.; Stepanova, Z.J.E. Effects of electrical and radio-frequency electromagnetic heating on the mass-transfer process during miscible injection for heavy-oil recovery. Fuels 2010, 25, 482-486. [CrossRef]

11. Adil, M.; Lee, K.; Mohd Zaid, H.; Ahmad Latiff, N.R.; Alnarabiji, M.S. Experimental study on electromagnetic-assisted $\mathrm{ZnO}$ nanofluid flooding for enhanced oil recovery (EOR). PLoS ONE 2018, 13, e0193518. [CrossRef]

12. Carrizales, M.A.; Lake, L.W.; Johns, R.T. Production improvement of heavy-oil recovery by using electromagnetic heating. In Proceedings of the SPE Annual Technical Conference and Exhibition, Denver, CO, USA, 21-24 September 2008.

13. Morte, M.; Bloom, E.; Huff, G.; Dean, J.; Hascakir, B. Factors Affecting Electromagnetic Wave Penetration in Heavy Oil Reservoirs. In Proceedings of the SPE Canada Heavy Oil Technical Conference, Calgary, AB, Canada, 13-14 March 2018.

14. Yahya, N.; Kashif, M.; Shafie, A.; Soleimani, H.; Zaid, H.M.; Latiff, N.R.A. Improved oil recovery by high magnetic flux density subjected to iron oxide nanofluids. J. Nano Res. 2013, 26, 89-99. [CrossRef]

15. Sahni, A.; Kumar, M.; Knapp, R.B. Electromagnetic Heating Methods for Heavy Oil Reservoirs; Lawrence Livermore National Lab.: Livermore, CA, USA, 2000. 
16. Kashif, M.; Yahya, N.; Zaid, H.M.; Shafie, A.; Jasamai, M.; Nasir, N.; Akhter, M.N. Oil Recovery by Using Electromagnetic Waves. J. Appl. Sci. 2011, 11, 1366-1370. [CrossRef]

17. Bera, A.; Babadagli, T. Status of electromagnetic heating for enhanced heavy oil/bitumen recovery and future prospects: A review. Appl. Energy 2015, 151, 206-226. [CrossRef]

18. Trautman, M.; Macfarlane, B. Experimental and numerical simulation results from a radio frequency heating test in native oil sands at the North Steepbank Mine. In Proceedings of the World Heavy Oil Congress, New Orleans, LA, USA, 5-7 March 2014; pp. 5-7.

19. Despande, S.R.; Wright, B.N.; Watt, A. Techniques for Installing Effective Solvent Extraction Incorporating Electromagnetic Heating ("ESEIEH") Completions. In Proceedings of the World Heavy Oil Congress, Edmonton, AB, Canada, 26-27 November 2019; pp. WHOC15-WHOC317.

20. Wise, S.; Patterson, C. Reducing Supply Cost With Eseieh ${ }^{\mathrm{TM}}$ Pronounced Easy. In Proceedings of the SPE Canada Heavy Oil Technical Conference, Calgary, AB, Canada, 7-9 June 2016.

21. Davletbaev, A.; Kovaleva, L.; Babadagli, T. Mathematical modeling and field application of heavy oil recovery by Radio-Frequency Electromagnetic stimulation. J. Petrol. Sci. Eng. 2011, 78, 646-653. [CrossRef]

22. Soleimani, H.; Yahya, N.; Latiff, N.R.A.; Zaid, H.M.; Demiral, B.; Amighian, J. Novel enhanced oil recovery method using $\mathrm{CO} 2+x \mathrm{Fe} 2+1-\mathrm{xFe} 3+2 \mathrm{O} 4$ as magnetic nanoparticles activated by electromagnetic waves. J. Nano Res. 2013, 26, 111-116. [CrossRef]

23. Jia, B.; Tsau, J.-S.; Barati, R. A review of the current progress of CO2 injection EOR and carbon storage in shale oil reservoirs. Fuel 2019, 236, 404-427. [CrossRef]

24. Thanh, N.T. Magnetic Nanoparticles: From Fabrication to Clinical Applications; CRC Press: Boca Raton, FL, USA, 2012.

25. Lee, K.; Adil, M.; Zaid, H.M.; Guan, B.H.; Soleimani, H.; Weis, M. Wettability, Interfacial Tension (IFT) and Viscosity Alteration of Nanofluids Under Electromagnetic (EM) Waves for Enhanced Oil Recovery (IFT) Applications. In Engineering Design Applications; Springer: Berlin, Germany, 2019; pp. 305-311.

26. Taheri-Shakib, J.; Shekarifard, A.; Naderi, H. The study of influence of electromagnetic waves on the wettability alteration of oil-wet calcite: Imprints in surface properties. J. Petrol. Sci. Eng. 2018, 168, 1-7. [CrossRef]

27. Hascakir, B.; Acar, C.; Akin, S. Microwave-assisted heavy oil production: An experimental approach. Energy Fuels 2009, 23, 6033-6039. [CrossRef]

28. Ovalles, C.; Vaca, P.; Okoniewski, M.; Dieckmann, G.; Pasalic, D.; Dunlavey, J. Numerical Simulation of Dielectric Heating in a Heavy Oil Reservoir Using a Shaped Dipole Antenna. In Proceedings of the SPE Canada Heavy Oil Technical Conference, Calgary, AB, Canada, 13-14 March 2018.

29. Santos, M.; Neto, A.; Mata, W.; Silva, J.J. New antenna modelling using wavelets for heavy oil thermal recovering methods. J. Petrol. Sci. Eng. 2011, 76, 63-75. [CrossRef]

30. Cassidy, N.J.; Jol, H. Electrical and magnetic properties of rocks, soils and fluids. In Ground Penetrating Radar Theory and Applications; Jol, H.M., Ed.; Elsevier: Amsterdam, The Netherlands, 2009; Volume 2.

31. Saeedfar, A.; Lawton, D.; Osadetz, K. Directional RF Heating for Heavy Oil Recovery Using Antenna Array Beam-Forming. In Proceedings of the SPE Canada Heavy Oil Technical Conference, Calgary, AB, Canada, 7-9 June 2016.

32. Stutzman, W.L.; Thiele, G.A. Antenna Theory and Design; John Wiley \& Sons: Hoboken, NJ, USA, 2012.

33. Wang, Z.; Gao, D.; Liu, K.; Tan, T.; Wang, Z.; Li, W. Study on Radio Frequency Heating Pattern of Heavy Oil Reservoir Based on Multi-Antenna Configuration. In Proceedings of the SPE International Heavy Oil Conference and Exhibition, Kuwait City, Kuwait, 10-12 December 2018.

34. Bera, A.; Babadagli, T.J. Effect of native and injected nano-particles on the efficiency of heavy oil recovery by radio frequency electromagnetic heating. J. Petrol. Sci. Eng. 2017, 153, 244-256. [CrossRef]

35. Mirabito, C.; Narayanan, A.; Pérez, D.; Stone, B. FEMLAB Model of a Coupled Electromagnetic-Thermal Boundary Value Problem; Worcester Polytechnic Institute: Worcester, MA, USA, 2005.

36. Shin, W.; Fan, S. Choice of the perfectly matched layer boundary condition for frequency-domain Maxwell's equations solvers. J. Comput. Phys. 2012, 231, 3406-3431. [CrossRef]

37. Gang, W.; Lin, A. COMSOL Multiphysics Engineering Practice and Theory Simulation: Multiphysics Numerical Analysis Technology; Electronic Industry Press: Beijing, China, 2013.

38. Xu, C.; Kang, Y.; You, Z.; Chen, M. Review on formation damage mechanisms and processes in shale gas reservoir: Known and to be known. J. Nat. Gas Sci. Eng. 2016, 36, 1208-1219. [CrossRef] 
39. Mousavi, M.; Hassanajili, S.; Rahimpour, M. Synthesis of fluorinated nano-silica and its application in wettability alteration near-wellbore region in gas condensate reservoirs. Appl. Surf. Sci. 2013, 273, $205-214$. [CrossRef]

40. Prodanovic, M.; Ryoo, S.; Rahmani, A.R.; Kuranov, R.V.; Kotsmar, C.; Milner, T.E.; Johnston, K.P.; Bryant, S.L.; Huh, C. Effects of magnetic field on the motion of multiphase fluids containing paramagnetic nanoparticles in porous media. In Proceedings of the SPE Improved Oil Recovery Symposium, Tulsa, OK, USA, 24-28 April 2010.

41. Bobrov, P.; Lapina, A.; Repin, A. Effect of the rock/water/air interaction on the complex dielectric permittivity and electromagnetic waves attenuation in water-saturated sandstones. In Proceedings of the PIERS Proceedings, Prague, Czech Republic, 6-9 July 2015; pp. 1877-1879.

42. Hu, Z.; Zhao, J.; Gao, H.; Nourafkan, E.; Wen, D. Transport and deposition of carbon nanoparticles in saturated porous media. Energies 2017, 10, 1151. [CrossRef]

43. Knight, R.J.; Nur, A. The dielectric constant of sandstones, $60 \mathrm{kHz}$ to $4 \mathrm{MHz}$. Geophysics 1987, 52, $644-654$. [CrossRef]

44. Fanchi, J.R. Feasibility of Near-Wellbore Heating by Electromagnetic Irradiation. SPE Adv. Technol. Ser. 1993, 1, 161-169. [CrossRef]

45. Sherman, M.M. Interpretation of dielectric permittivity measurements in the 20-to 50-MHz frequency range. SPE Form. Eval. 1990, 5, 76-80. [CrossRef]

46. Slyusar, V.I. 60 years of electrically small antennas theory. In Proceedings of the 2007 6th International Conference on Antenna Theory and Techniques, Sevastopol, Ukraine, 17-21 September 2007; pp. 116-118.

47. Li, R.; Li, B.; Du, G.; Sun, X.; Sun, H. A Compact Broadband Antenna with Dual-Resonance for Implantable Devices. Micromachines 2019, 10, 59. [CrossRef]

48. Idris, F.M.; Hashim, M.; Abbas, Z.; Ismail, I.; Nazlan, R.; Ibrahim, I.R. Recent developments of smart electromagnetic absorbers based polymer-composites at gigahertz frequencies. J. Magn. Magn. Mater. 2016, 405, 197-208. [CrossRef]

49. Lalatonne, Y.; Richardi, J.; Pileni, M. Van der Waals versus dipolar forces controlling mesoscopic organizations of magnetic nanocrystals. Nat. Mater. 2004, 3, 121. [CrossRef]

50. Faure, B.; Salazar-Alvarez, G.; Bergström, L. Hamaker constants of iron oxide nanoparticles. Langmuir 2011, 27, 8659-8664. [CrossRef] 